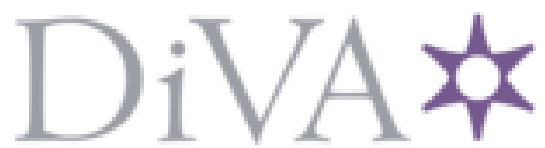

http://www.diva-portal.org

Preprint

This is the submitted version of a paper presented at 19th International Conference on Electromagnetics in Advanced Applications, ICEAA 2017, Verona, Italy, 11 September 2017 through 15 September 2017.

Citation for the original published paper:

Malmström, J., Jonsson, L. (2017)

An Effective Method for Antenna Placement on Platforms Based on the Reaction

Theorem

In: Proceedings of the 2017 19th International Conference on Electromagnetics in Advanced Applications, ICEAA 2017, 8065579 (pp. 1547-1550). Institute of Electrical and Electronics Engineers (IEEE)

https://doi.org/10.1109/ICEAA.2017.8065579

N.B. When citing this work, cite the original published paper.

Permanent link to this version:

http://urn.kb.se/resolve?urn=urn:nbn:se:kth:diva-215193 


\section{An Effective Method for Antenna Placement on Platforms Based on the Reaction Theorem}

\author{
J. Malmström*
}

\begin{abstract}
-
Simulations for antenna placement on large platforms are computational costly. We here suggest a very efficient method for antenna placement optimization of low-scattering antennas. The method utilizes the reaction theorem to calculate the mutual impedance between the antennas based on precomputed electromagnetic fields. The method needs only one full-platform simulation together with postprocessing of field data to estimate the coupling for all antenna positions in a given region. In the tested case, we see a speed-up with a factor of 2000 , compared to full-wave simulations of every antenna position to be evaluated.
\end{abstract}

\section{INTRODUCTION}

When designing platforms with RF functionality, antenna placement is an important consideration to ensure electromagnetic compatibility (EMC). In antenna placement studies, the mutual coupling between antennas is a key quantity [1]. To ensure EMC, the mutual coupling should be low.

The mutual coupling can be determined from a full-wave simulation with the antennas installed on the platform. The mutual coupling usually depends strongly on the platform geometry and the antenna positions on the platform. In general, a simulation has to be performed for each antenna position to be evaluated. For large platforms, each full-wave simulation usually takes a considerable amount of time, which limits the number of antenna positions that are practically possible to evaluate.

Other methods to calculate the mutual coupling between antennas on large platforms has been proposed and investigated, see e.g. $[2,3,4]$.

The reaction theorem can be used to determine mutual impedance between antennas $[5,6,7]$. It has been used in several applications recently, see e.g. $[8,9,10]$, also for antennas installed on platforms.

The mutual impedance and the mutual coupling between antennas are closely related [11], in particular for weak coupling cases. Therefore, we can use the mutual impedance as a parameter for optimization instead of mutual coupling.

\footnotetext{
* Saab Surveillance, Stockholm, Sweden, and Electromagnetic Theory, KTH Royal Institute of Technology, Stockholm, Sweden

$\dagger$ Electromagnetic Theory, KTH Royal Institute of Technology, Stockholm, Sweden
}

\author{
B. L. G. Jonsson ${ }^{\dagger}$
}

(a)

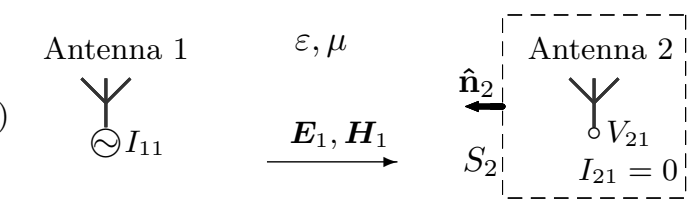

(b)

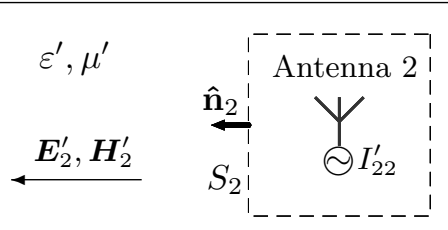

Figure 1: (a) The fields $\boldsymbol{E}_{1}, \boldsymbol{H}_{1}$ generated by Antenna 1, and (b) the fields $\boldsymbol{E}_{2}^{\prime}, \boldsymbol{H}_{2}^{\prime}$ generated by Antenna 2 in an alternative environment.

\section{THEORY}

The generalized reaction theorem can be formulated in terms of the mutual impedance $Z_{21}$ between two antennas [6],

$$
Z_{21}=\frac{-1}{I_{11} I_{22}^{\prime}} \oint_{S_{2}}\left(\boldsymbol{E}_{2}^{\prime} \times \boldsymbol{H}_{1}-\boldsymbol{E}_{1} \times \boldsymbol{H}_{2}^{\prime}\right) \cdot \hat{\mathbf{n}}_{2} \mathrm{~d} S
$$

where the integration surface $S_{2}$, with outward pointing normal $\hat{\mathbf{n}}_{2}$, encloses Antenna 2 but not Antenna 1, see Fig. 1. Antenna 1 generates the fields $\boldsymbol{E}_{1}, \boldsymbol{H}_{1}$ when excited with a current $I_{11}$, as in Fig. 1(a). Antenna 2 generates the fields $\boldsymbol{E}_{2}^{\prime}, \boldsymbol{H}_{2}^{\prime}$ when excited with a current $I_{22}^{\prime}$, as in Fig. 1(b). The excitation currents $I_{11}, I_{22}^{\prime}$ are measured on each of the two antenna terminals. For (1) to hold, the terminal in antenna 2 must be open circuit when Antenna 1 transmits.

The generalized reaction theorem (1) allows the structure outside the integration surface to be changed when transmission with the antenna enclosed by the integration surface [6], as depicted in Fig. 1. Here, we utilize this to choose the exterior to $S_{2}$ to be empty.

We decompose $\boldsymbol{E}_{1}, \boldsymbol{H}_{1}$ as a sum of multiple scattering components as illustrated in Fig. 2. The decomposition together with the Silver-Müller radiation condition allows (1) to be reduced to [12],

$Z_{21}=\frac{-1}{I_{11} I_{22}^{\prime}} \oint_{S_{2}}\left(\boldsymbol{E}_{2}^{\prime} \times \boldsymbol{H}_{1}^{+}-\boldsymbol{E}_{1}^{+} \times \boldsymbol{H}_{2}^{\prime}\right) \cdot \hat{\mathbf{n}}_{2} \mathrm{~d} S$. 


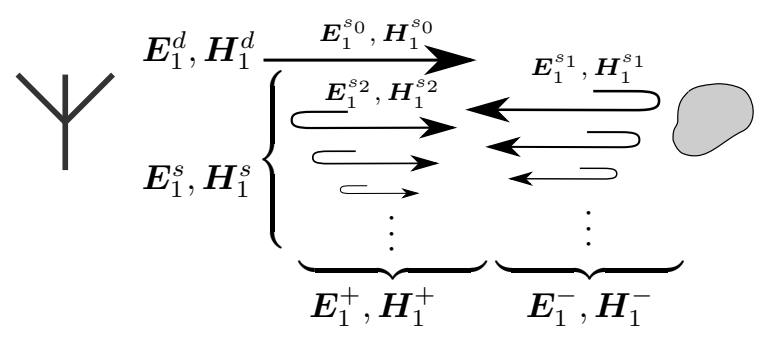

Figure 2: Separation of the fields $\boldsymbol{E}_{1}, \boldsymbol{H}_{1}$ generated by an antenna (left) and scattered on an arbitrary object (right) into either direct $\boldsymbol{E}_{1}^{d}, \boldsymbol{H}_{1}^{d}$ and scattered fields $\boldsymbol{E}_{1}^{s}, \boldsymbol{H}_{1}^{s}$, or into outgoing $\boldsymbol{E}_{1}^{+}, \boldsymbol{H}_{1}^{+}$and incoming fields $\boldsymbol{E}_{1}^{-}, \boldsymbol{H}_{1}^{-}$.

An approximation of the outgoing fields $\boldsymbol{E}_{1}^{+}, \boldsymbol{H}_{1}^{+}$, can be formed by neglecting multiple scattering and only taking the direct components into account,

$$
\begin{aligned}
& \boldsymbol{E}_{1}^{+} \approx \boldsymbol{E}_{1}^{d}, \\
& \boldsymbol{H}_{1}^{+} \approx \boldsymbol{H}_{1}^{d} .
\end{aligned}
$$

This approximations introduce the errors $\delta \boldsymbol{E}_{1}, \delta \boldsymbol{H}_{1}$

$$
\begin{gathered}
\delta \boldsymbol{E}_{1}=\boldsymbol{E}_{1}^{+}-\boldsymbol{E}_{1}^{d}=\sum_{n=2,4,6, \ldots}^{\infty} \boldsymbol{E}^{s_{n}} \\
\delta \boldsymbol{H}_{1}=\boldsymbol{H}_{1}^{+}-\boldsymbol{H}_{1}^{d}=\sum_{n=2,4,6, \ldots}^{\infty} \boldsymbol{H}^{s_{n}} .
\end{gathered}
$$

Note that the sums start at $n=2$ since the first order scattering $\boldsymbol{E}^{s_{1}}, \boldsymbol{H}^{s_{1}}$ on Antenna 2 do not affect the error. This is understood by the fact that $\boldsymbol{E}_{1}^{-}, \boldsymbol{H}_{1}^{-}$is not included in (2). The lowest order scattering component that contribute to the error is $\boldsymbol{E}_{1}^{s_{2}}, \boldsymbol{H}_{1}^{s_{2}}$. For low-scattering antennas, the errors $\delta \boldsymbol{E}_{1}, \delta \boldsymbol{H}_{1}$ will be small.

Mutual impedance and mutual coupling are closely related [11]. In a two-antenna system, the mutual coupling $S_{21}$ is approximately proportional to the mutual impedance $Z_{21}$, if $\left|Z_{21} Z_{12}\right| \ll$ $\left|Z_{11} Z_{22}\right|$. Therefore, we will consider an optimization on mutual impedance $Z_{21}$ in this paper.

The reaction theorem, when used for analysing each antenna in separation, as in Fig. 1, has similarities with domain decomposition methods, described in e.g. [13].

\section{METHOD}

Assume that we have a platform with one antenna installed and want to find the best position for an additional antenna. Further, assume that "best" can be quantified in terms of the smallest mutual impedance between the two antennas. ${ }^{1}$ An-

\footnotetext{
${ }^{1}$ An additional criteria should describe the radiation requirements for the new antenna, e.g. requirements on the
}

(a)

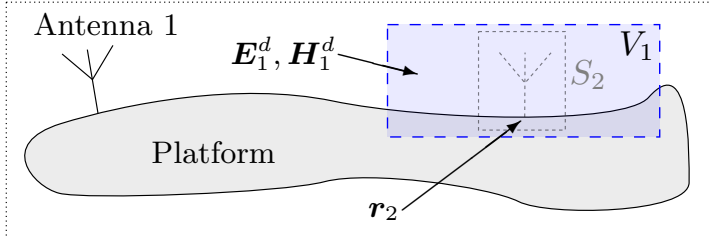

(b)

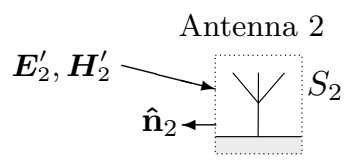

Figure 3: (a) The complete environment including the platform and the installed Antenna 1 and (b) the alternative environment including only Antenna 2 and the structure within the surface $S_{2}$.

tenna 1 is mounted at a fixed position and the possible positions for Antenna 2 are restricted to a region $V_{1}$ on the platform. The platform is electrically large, whereas the size of the antennas is comparable to a wavelength.

The mutual impedance $Z_{21}$ between the antennas will change for different positions $\boldsymbol{r}_{2}$ of Antenna 2. With the above assumptions, the best location $\boldsymbol{r}_{\mathrm{opt}}$ for Antenna 2 can be formulated as an optimization problem.

$$
\boldsymbol{r}_{\mathrm{opt}}=\arg \min _{\boldsymbol{r}_{2}}\left|Z_{21}\left(\boldsymbol{r}_{2}\right)\right|
$$

The reaction theorem (2) can be used to reformulate (7) into

$$
\arg \min _{\boldsymbol{r}_{2}}\left|\frac{\oint_{S_{2}}\left(\boldsymbol{E}_{2}^{\prime} \times \boldsymbol{H}_{1}^{+}-\boldsymbol{E}_{1}^{+} \times \boldsymbol{H}_{2}^{\prime}\right) \cdot \hat{\mathbf{n}}_{2} \mathrm{~d} S}{I_{11} I_{22}^{\prime}}\right| .
$$

The fields $\boldsymbol{H}_{1}^{+}, \boldsymbol{E}_{1}^{+}$depend on the position $\boldsymbol{r}_{2}$ of Antenna 2 (and the enclosing surface $S_{2}$ ). Hence, we can calculate the optimal antenna position with only the pre-computed electromagnetic fields on a closed surface $S_{2}$ separating the antennas in two disjoint regions. The currents, $I_{11}$ and $I_{22}^{\prime}$, are known from when the fields where generated. The excitation current $I_{22}^{\prime}$ is independent of the position $r_{2}$ of Antenna 2. The current $I_{11}$ is also independent of $r_{2}$ if neglecting scattering on Antenna 2, see (3)-(4). The optimization problem simplifies to

$$
\arg \min _{\boldsymbol{r}_{2}}\left|\oint_{S_{2}}\left(\boldsymbol{E}_{2}^{\prime} \times \boldsymbol{H}_{1}^{d}-\boldsymbol{E}_{1}^{d} \times \boldsymbol{H}_{2}^{\prime}\right) \cdot \hat{\mathbf{n}}_{2} \mathrm{~d} S\right| .
$$

The fields $\boldsymbol{E}_{1}^{d}, \boldsymbol{H}_{1}^{d}$ are determined in $V_{1}$ without the presence of Antenna 2, see Fig. 3(a), and can hence

far-fields pattern or reflection parameter thresholds. We assume that the radiation criteria are handled separate from the mutual impedance criteria. 


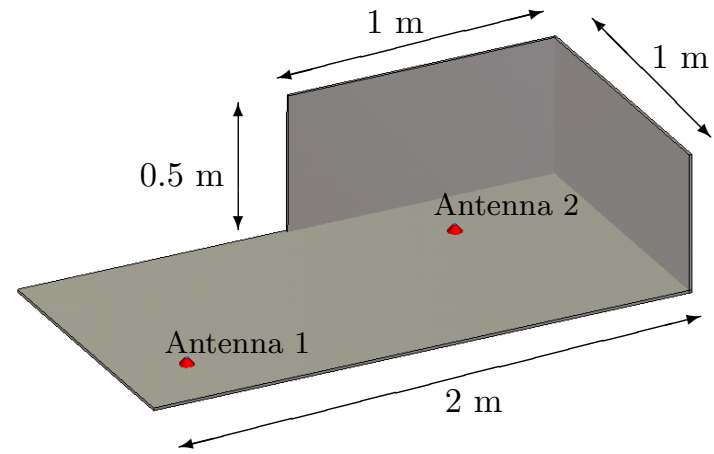

Figure 4: The model used; a PEC platform with a $90^{\circ}$ corner, Antenna 1 (fixed), and Antenna 2 (to be installed at some position).

be computed once. The integral in (9) selects a subset of $\boldsymbol{E}_{1}^{d}, \boldsymbol{H}_{1}^{d}$ in $V_{1}$ based on the position $\boldsymbol{r}_{2}$ of $S_{2}$.

The suggested method is summarized as follows:

1. Determine the fields $\boldsymbol{E}_{1}^{d}, \boldsymbol{H}_{1}^{d}$ in the volume $V_{1}$ by solving the full-platform EM problem without Antenna 2, see Fig. 3(a).

2. Determine the fields $\boldsymbol{E}_{2}^{\prime}, \boldsymbol{H}_{2}^{\prime}$ on the surface $S_{2}$ in the second environment with only Antenna 2 present, see Fig. 3(b).

3. Use the fields $\boldsymbol{E}_{1}^{d}, \boldsymbol{H}_{1}^{d}$ and $\boldsymbol{E}_{2}^{\prime}, \boldsymbol{H}_{2}^{\prime}$ from steps 1-2 to evaluate the expression in (9). Repeat for all positions $\boldsymbol{r}_{2}$ of Antenna 2 (and the enclosing surface $S_{2}$ ) where $\boldsymbol{E}_{1}^{d}, \boldsymbol{H}_{1}^{d}$ are known.

4. Find the smallest value calculated in Step 3. The corresponding position $\boldsymbol{r}_{2}$ is the optimal placement for Antenna 2.

The time for Step 1 depends on the size of the platform. On large platforms, it is by far the most time consuming step. The time for Step 3 depends on the number of samples on the integration surface $S_{2}$.

\section{NUMERICAL RESULTS}

We illustrate the here proposed method for finding the optimal antenna position by investigating the platform depicted in Fig. 4. The platform is designed to give strong backscattering. Antenna 1 and 2 are $25 \mathrm{~mm}$ monopoles excited at $3 \mathrm{GHz}$. At this frequency, the platform size is $20 \lambda \times 10 \lambda \times 5 \lambda$.

The volume $V_{1}$ is $1 \mathrm{~m} \times 1 \mathrm{~m} \times 50 \mathrm{~mm}$. The integration surface $S_{2}$ is a Cartesian box enclosing Antenna 2. The volume enclosed by $S_{2}$ is $100 \mathrm{~mm}$ $\times 100 \mathrm{~mm} \times 50 \mathrm{~mm}$, with the smallest dimension perpendicular to the platform. The sample spacing in the volume $V_{1}$ and on the surface $S_{2}$ is $2.5 \mathrm{~mm}$.

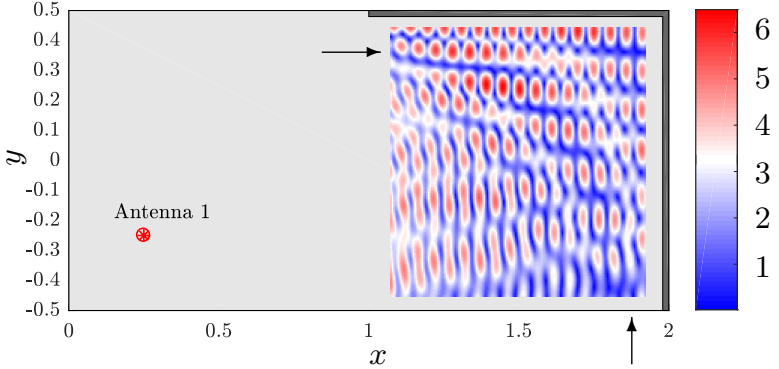

Figure 5: Mutual impedance magnitude $\left|Z_{21}(x, y)\right|$ in $\Omega$ for 30430 different positions $\boldsymbol{r}_{2}=(x, y)$ of Antenna 2 on the platform. The optimal position $\boldsymbol{r}_{\mathrm{opt}}$, according to $(9)$, is marked with arrows.

To evaluate the integral in (9), we sum over 6402 discrete points on $S_{2}$ for each position $\boldsymbol{r}_{2}$.

The position $\boldsymbol{r}_{2}$ of Antenna 2 is swept in $x, y$ within $V_{1}$, as illustrated by the $2 \mathrm{D}$ mutual impedance map in Fig. 5. Each position on the color coded map reflects the mutual impedance between Antenna 1 and Antenna 2 for that specific position $\boldsymbol{r}_{2}$ of Antenna 2 .

For the tested case, the time $t_{0}$ to calculate the mutual impedance in $n$ antenna positions with a full-wave simulation (FIT in CST Microwave Studio (MWS) with 12 mesh cells per wavelength) is

$$
t_{0}(n)=n \cdot 400 \mathrm{~s} .
$$

As a comparison, the time $t_{1}$ with the suggested method is

$$
t_{1}(n)=\underbrace{410 \mathrm{~s}}_{1 .}+\underbrace{20 \mathrm{~s}}_{2 .}+\underbrace{n \cdot 0.2 \mathrm{~s}}_{3 .}+\underbrace{0.001 \mathrm{~s}}_{4 .},
$$

where each term corresponds to the time for the four steps ${ }^{2}$ described in Sec. 3.

The incremental time to evaluate one antenna position is $400 \mathrm{~s}$ with the full-wave method and $0.2 \mathrm{~s}$ with the suggested method. That is a speed-up with a factor of 2000 . To calculate the $30430 \mathrm{mu}-$ tual impedances in Fig. 5 would take $t_{0}(30430) \approx$ 4.7 months with full-wave simulations, compared to $t_{1}(30430) \approx 1.7$ hours with the suggested method.

The accuracy of the method is verified by comparing with full-wave simulations of $Z_{21}$ using CST MWS for a subset of the evaluated Antenna 2 positions, see Fig. 6. Neglecting scattering from Antenna 2 introduces small discrepancies in $\left|Z_{21}\right|$. However, the position of the minimum of $\left|Z_{21}\right|$ agrees excellent, which indicate that the method correctly finds the optimal antenna position.

\footnotetext{
${ }^{2}$ The dependence of $n$ in Step 4. is neglected.
} 


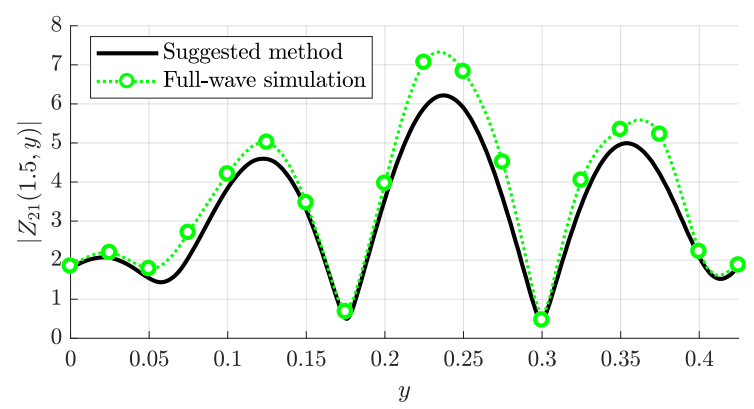

Figure 6: Mutual impedance magnitude $\left|Z_{21}\right|$ for Antenna 2 positions $x=1.5 \mathrm{~m}, y=[0,0.425] \mathrm{m}$.

\section{DISCUSSION AND CONCLUSIONS}

In this paper, we suggest an efficient method to find the optimal antenna position on large platforms. The method uses the reaction theorem to formulate an optimization problem involving only electromagnetic fields generated by the two antennas. The fields can be obtained in two full-wave simulation; one with the platform present, and one with only the new antenna. The optimization is performed as a post-processing step based on EM fields, e.g. from a commercial EM simulation software. It does not require any internal data from the full-wave simulation. The suggested method correctly finds the optimal antenna position for lowscattering antennas.

The method significantly decreases the computational time for antenna placement studies involving electrically large platforms. In the studied case, we see a speed-up with a factor of 2000 , compared to running a full-wave simulation for each antenna position.

Additional antenna types can be evaluated using fields from one additional simulation with the new antenna type in isolation. The same pre-computed fields with the platform present can be re-used.

\section{Acknowledgments}

The authors acknowledge Saab Surveillance for funding. LJ is grateful for funding from SSF/AM130011 and Vinnova/ChaseOn through iAA.

\section{References}

[1] T. M. Macnamara, Introduction to Antenna Placement and Installation. John Wiley \& Sons, 2010.

[2] A. D. Yaghjian, "Efficient Computation of Antenna Coupling and Fields Within the Nearfield Region," IEEE Trans. Antennas Propag., vol. 30, no. 1, pp. 113-128, 1982.

[3] H. Zhao, et al., "A linear model of transmission coefficients for placement of monopole antennas on electrically large cylindrical surfaces," in URSI Gen. Assem. Sci. Symp., 2014.

[4] J. Malmström, et al., "Approximate Methods to Determine the Isolation between Antennas on Vehicles," in 2016 IEEE Antennas Propag. Soc. Int. Symp., pp. 131-132, 2016.

[5] V. H. Rumsey, "Reaction concept in electromagnetic theory," Phys. Rev., vol. 94, no. 6, pp. 1483-1491, 1954.

[6] J. H. Richmond, "A reaction theorem and its application to antenna impedance calculations," Antennas Propagation, IRE Trans., vol. 9, no. 6, pp. 515-520, 1961.

[7] C. A. Balanis, Antenna Theory Analysis and Design. John Wiley \& Sons, 3rd ed., 2005.

[8] H. Wang et al., "Estimating radio-frequency interference to an antenna due to near-field coupling using decomposition method based on reciprocity," IEEE Trans. Electromagn. Compat., vol. 55, no. 6, pp. 1125-1131, 2013.

[9] L. Li et al., "Radiation Noise Source Modeling and Application in Near-Field Coupling Estimation," IEEE Trans. Electromagn. Compat., vol. 58, no. 4, pp. 1314-1321, 2016.

[10] C. Craeye, "On the transmittance between OAM antennas," IEEE Trans. Antennas Propag., vol. 64, no. 1, pp. 336-339, 2016.

[11] D. M. Pozar, Microwave Engineering. John Wiley \& Sons, 4th ed., 2005.

[12] J. Malmström, et al., "On the Reaction Theorem for Scattered Fields," in preparation, 2017.

[13] A. Toselli and O. Widlund, Domain Decomposition Methods - Algorithms and Theory. Springer-Verlag, 1st ed., 2005. 